\title{
Two-electron coherence and its measurement in electron quantum optics
}

\author{
É. Thibierge ${ }^{1}$, D. Ferraro ${ }^{1,2,3}$, B. Roussel ${ }^{1,4}$, C. Cabart ${ }^{5}$, A. Marguerite ${ }^{5}$, G. Fève ${ }^{5}$, and P. Degiovanni ${ }^{1}$ \\ (1) Université de Lyon, Fédération de Physique A.-M. Ampère, \\ CNRS - Laboratoire de Physique - Ecole Normale Supérieure de Lyon, \\ 46 Allée d'Italie, 69364 Lyon Cedex 07, France \\ (2) Aix Marseille Université, CNRS, CPT, UMR 7332, 13288 Marseille, France \\ (3) Université de Toulon, CNRS, CPT, UMR 7332, 83957 La Garde, France \\ (4) Université Claude Bernard Lyon 1, \\ $43 \mathrm{Bd} d u 11$ Novembre 1918 , \\ 69622 Villeurbanne Cedex, France and \\ (5) Laboratoire Pierre Aigrain, Ecole Normale Supérieure-PSL Research University, \\ CNRS, Université Pierre et Marie Curie-Sorbonne Universités, \\ Université Paris Diderot-Sorbonne Paris Cité, 24 rue Lhomond, 75231 Paris Cedex 05, France.
}

\begin{abstract}
Engineering and studying few-electron states in ballistic conductors is a key step towards understanding entanglement in quantum electronic systems. In this Letter, we introduce the intrinsic two-electron coherence of an electronic source in quantum Hall edge channels and relate it to twoelectron wavefunctions and to current noise in an Hanbury Brown-Twiss interferometer. Inspired by the analogy with photon quantum optics, we propose to measure the intrinsic two-electron coherence of a source using low-frequency current correlation measurements at the output of a Franson interferometer. To illustrate this protocol, we discuss how it can distinguish between a time-bin entangled pure state and a statistical mixture of time shifted electron pairs.
\end{abstract}

PACS numbers: 73.23.-b,73.43.-f,71.10.Pm, 73.43.Lp

Keywords: quantum Hall effect, quantum transport, electronic coherence, entanglement

The initial development of electron quantum optics [1] has focused on single electron coherence [2, 3] in ballistic quantum conductors through Mach-Zehnder interferometry [4 6] and electronic distribution function measurement [7, 8]. With the availability of single electron sources 9-13, Hanbury Brown-Twiss (HBT) [14 and Hong-Ou-Mandel (HOM) experiments [15] have been demonstrated. These two-particle interference experiments are important milestones towards single electron tomography [2, mirroring optical homodyne tomography [16]. Recent experimental progresses [17] suggest that technology has reached the point where single electron coherence can be measured using signal processing strategies [18], thus opening the way to quantitative studies of electronic decoherence [19, 20] and decoherence protection techniques [21, 22,

The new frontier for electron quantum optics is now the emergence of many-body physics in quantum nanoelectronics. Beyond understanding single electron decoherence, electron quantum optics gives a unique and new insight on the build up of quantum correlations in a coherent conductor as a result of strong Coulomb interactions and fermionic statistics. For example, studying interaction induced decoherence of engineered few electron excitations (among which the charge- $n$ Leviton 23, 24) is essential for understanding the interplay of interaction effects among the $n$ particles and many-body effects involving all electrons present in the Fermi sea such as decoherence an relaxation. By encoding all the information on $n$-electronic wave functions present in the electronic fluid, higher order electronic coherences [25] defined by analogy with their photonic counterparts [26] are the appropriate quantities to tackle these problems. Moreover, because entanglement criteria can be expressed in terms of many-body correlators [27, higher electronic coherences will also be instrumental in quantifying entanglement in the electronic many-body fluid 28. From a broader perspective, understanding higher-order coherences in presence of a Fermi sea is also relevant for quantum transport simulations with fermionic cold atom systems [29, 30] and atomic HOM experiments [31.

In this Letter, we make a step in this direction by discussing the recently introduced two-electron coherence 25, an essential quantity to quantify two-particle entanglement beyond the orbital point of view [32 36], that is, in the time and energy domains. In this perspective, we show how to define the intrinsic two-electron coherence emitted by an electron source and discuss how it encodes the information on two-electron wavefunctions. We then propose to access it through current correlations in a Franson interferometer [37] which has also been proposed to study Cooper pair coherence [38]. This naturally extends single electron coherence measurement through average current in Mach-Zehnder interferometry [39].

By analogy with quantum optics [26, 40, two-electron coherence is defined as 25:

$$
\mathcal{G}_{\rho}^{(2 \mathrm{e})}\left(1,2 \mid 1^{\prime}, 2^{\prime}\right)=\operatorname{Tr}\left[\psi(2) \psi(1) \rho \psi^{\dagger}\left(1^{\prime}\right) \psi^{\dagger}\left(2^{\prime}\right)\right]
$$

where $\psi$ and $\psi^{\dagger}$ are field operators for the electron fluid, $\rho$ its density operator and $1=\left(x_{1}, t_{1}\right), 2=\left(x_{2}, t_{2}\right), 1^{\prime}=$ $\left(x_{1}^{\prime}, t_{1}^{\prime}\right)$ and $2^{\prime}=\left(x_{2}^{\prime}, t_{2}^{\prime}\right)$ four space-time coordinates. 
Activating a source $(S)$ generating excitations on top of the Fermi sea alters all electronic coherences. A sourceintrinsic contribution to the total single electron coherence $\mathcal{G}_{S}^{(\mathrm{e})}\left(1 \mid 1^{\prime}\right)=\operatorname{Tr}\left[\psi(1) \rho_{S} \psi^{\dagger}\left(1^{\prime}\right)\right]$ can be defined [3, 41] by substracting the Fermi sea contribution $\mathcal{G}_{F}^{(\mathrm{e})}$ :

$$
\Delta \mathcal{G}_{S}^{(\mathrm{e})}\left(1 \mid 1^{\prime}\right)=\mathcal{G}_{S}^{(\mathrm{e})}\left(1 \mid 1^{\prime}\right)-\mathcal{G}_{F}^{(\mathrm{e})}\left(1 \mid 1^{\prime}\right) .
$$

To identify the intrinsic second order coherence of the source from the full $\mathcal{G}_{S}^{(2 \mathrm{e})}$, we must consider all processes contributing to the co-detection of two electrons, depicted on Fig. 1. The total two-electron coherence is the sum of (a) the two-electron coherence of the Fermi sea; (b) terms describing classical coincidences in which one electron comes from the Fermi sea and the other one from the source; (c) quantum exchange contributions involving two-particle interferences between the source and the Fermi sea; (d) the remaining term that defines intrinsic two-electron coherence of the source $\Delta \mathcal{G}_{S}^{(2 \mathrm{e})}\left(1,2 \mid 1^{\prime}, 2^{\prime}\right)$ :

$$
\begin{aligned}
\mathcal{G}_{S}^{(2 \mathrm{e})} & \left(1,2 \mid 1^{\prime}, 2^{\prime}\right)=\mathcal{G}_{F}^{(2 \mathrm{e})}\left(1,2 \mid 1^{\prime}, 2^{\prime}\right) \\
& +\mathcal{G}_{F}^{(\mathrm{e})}\left(1 \mid 1^{\prime}\right) \Delta \mathcal{G}_{S}^{(\mathrm{e})}\left(2 \mid 2^{\prime}\right)+\mathcal{G}_{F}^{(\mathrm{e})}\left(2 \mid 2^{\prime}\right) \Delta \mathcal{G}_{S}^{(\mathrm{e})}\left(1 \mid 1^{\prime}\right) \\
& -\mathcal{G}_{F}^{(\mathrm{e})}\left(1 \mid 2^{\prime}\right) \Delta \mathcal{G}_{S}^{(\mathrm{e})}\left(2 \mid 1^{\prime}\right)-\mathcal{G}_{F}^{(\mathrm{e})}\left(2 \mid 1^{\prime}\right) \Delta \mathcal{G}_{S}^{(\mathrm{e})}\left(1 \mid 2^{\prime}\right) \\
& +\Delta \mathcal{G}_{S}^{(2 \mathrm{e})}\left(1,2 \mid 1^{\prime}, 2^{\prime}\right) .
\end{aligned}
$$

The definition of $\Delta \mathcal{G}_{S}^{(2 \mathrm{e})}$ given by Eq. (3) is the second in a hierarchy of definitions for the $n$-electron excess coherence with respect to a reference situation, here the Fermi sea. All of them make sense even in the presence of interactions since their definition does not rely on Wick theorem.

The physical meaning of intrinsic two-electron coherence can be illustrated by a few examples. We consider fixed positions for detectors so that only time variables are mentioned. Interactions are neglected. First, we consider an ideal single electron source emitting a single electronic excitation in a wavepacket $\varphi$. Using Wick theorem, the excess single electron coherence is found to be $\Delta \mathcal{G}_{S}^{(\mathrm{e})}\left(t \mid t^{\prime}\right)=\varphi(t) \varphi^{*}\left(t^{\prime}\right)$ whereas $\Delta \mathcal{G}_{S}^{(2 \mathrm{e})}\left(t_{1}, t_{2} \mid t_{1}^{\prime}, t_{2}^{\prime}\right)$ vanishes as expected from the analogy with quantum optics. An ideal two-electron source emitting a two-electron excitation above the Fermi sea $\psi^{\dagger}\left[\varphi_{a}\right] \psi^{\dagger}\left[\varphi_{b}\right]|F\rangle$, where $\varphi_{a, b}$ are two orthogonal normalized electronic wavepackets generates:

$$
\begin{aligned}
& \Delta \mathcal{G}_{S}^{(\mathrm{e})}\left(t \mid t^{\prime}\right)=\varphi_{a}(t) \varphi_{a}^{*}\left(t^{\prime}\right)+\varphi_{b}(t) \varphi_{b}^{*}\left(t^{\prime}\right), \\
& \Delta \mathcal{G}_{S}^{(2 \mathrm{e})}\left(t_{1}, t_{2} \mid t_{1}^{\prime}, t_{2}^{\prime}\right)=\Phi_{a b}\left(t_{1}, t_{2}\right) \Phi_{a b}^{*}\left(t_{1}^{\prime}, t_{2}^{\prime}\right) ;
\end{aligned}
$$

where $\Phi_{a b}$ is the Slater determinant built from $\varphi_{a}$ and $\varphi_{b}$,

$$
\Phi_{a b}\left(t_{1}, t_{2}\right)=\varphi_{a}\left(t_{1}\right) \varphi_{b}\left(t_{2}\right)-\varphi_{b}\left(t_{1}\right) \varphi_{a}\left(t_{2}\right) .
$$

Therefore, $\Delta \mathcal{G}_{S}^{(2 \mathrm{e})}$ gives access to the two-particle wave function emitted by the source, extending at the two particle level the relation of $\Delta \mathcal{G}_{S}^{(\mathrm{e})}$ to single particle wavefunctions [2]. The case of an electron train built from

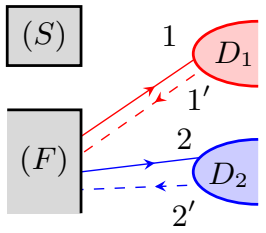

(a) Fermi sea.

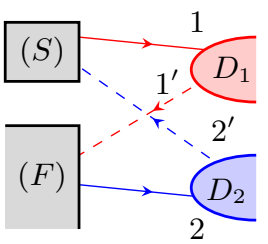

(c) Quantum exchange.

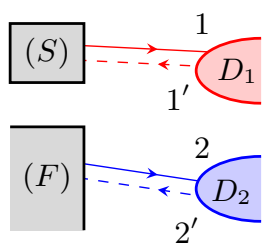

(b) Classical correlations.

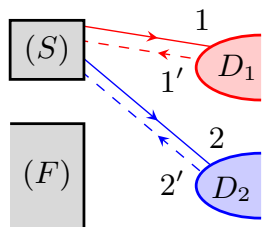

(d) Source intrinsic.
FIG. 1: (Color online) Contributions to the two-electron detection probability by detectors $D_{1}$ and $D_{2}$. Full lines represent direct probability amplitudes whereas dotted lines represent complex conjugated ones. The electronic system is decomposed into the Fermi sea $(F)$ and a source $(S)$ emitting electron and/or hole excitations. The two-electron detection probability contains four types of contributions.

mutually orthogonal wavepackets extends Eq. 4b into a sum involving all pairs of electronic excitations,

$$
\Delta \mathcal{G}^{(2 \mathrm{e})}\left(t_{1}, t_{2} \mid t_{1}^{\prime}, t_{2}^{\prime}\right)=\sum_{\text {pairs }\{i, j\}} \Phi_{i j}\left(t_{1}, t_{2}\right) \Phi_{i j}^{*}\left(t_{1}^{\prime}, t_{2}^{\prime}\right) .
$$

The diagonal part $\Delta \mathcal{G}_{S}^{(2 \mathrm{e})}\left(t_{1}, t_{2} \mid t_{1}, t_{2}\right)$ of the intrinsic twoelectron coherence encodes the two-particle time correlations of the electron flow. By analogy with quantum optics, we define the degree of second order electronic coherence of the source by

$$
g_{S}^{(2 \mathrm{e})}\left(t_{1}, t_{2}\right)=\frac{\Delta \mathcal{G}_{S}^{(2 \mathrm{e})}\left(t_{1}, t_{2} \mid t_{1}, t_{2}\right)}{\Delta \mathcal{G}_{S}^{(\mathrm{e})}\left(t_{1} \mid t_{1}\right) \Delta \mathcal{G}_{S}^{(\mathrm{e})}\left(t_{2} \mid t_{2}\right)} .
$$

Statistical independence of the detection events at $t_{1}$ and $t_{2}$ corresponds to $g_{S}^{(2 \mathrm{e})}\left(t_{1}, t_{2}\right)=1$ whereas antibunching is associated with $g_{S}^{(2 \mathrm{e})}\left(t_{1}, t_{2}\right)<1$ and bunching with $g_{S}^{(2 \mathrm{e})}\left(t_{1}, t_{2}\right)>1$.

As an example, Fig. 2 presents $\Delta \mathcal{G}_{S}^{(2 \mathrm{e})}\left(t_{1}, t_{2} \mid t_{1}, t_{2}\right)$ for an ideal source emitting a Slater determinant built from three Landau excitations 20] of duration $\tau_{\mathrm{e}}$ and energy $\hbar \omega_{\mathrm{e}}\left[53\right.$. separated by $\Delta t=3 \tau_{\mathrm{e}}$. Correlations are maximal at the times associated with two electron emissions and vanish around the diagonal $t_{1} \simeq t_{2}$ as expected from Pauli principle.

In the context of electron quantum optics, two-electron properties can be accessed through current noise measurement. But the current noise 42 , 43 of a source contains contributions from terms (3b) and (3c). However, the HBT setup [14, 44 provides a more direct access 


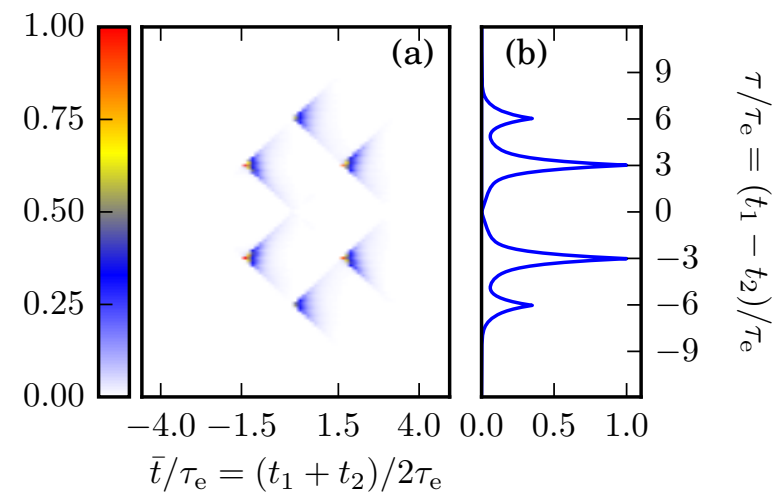

FIG. 2: (Color online) (a) Normalized density plot of $\Delta \mathcal{G}_{S}^{(2 \mathrm{e})}\left(t_{1}, t_{2} \mid t_{1}, t_{2}\right)$ as a function of $\bar{t}=\left(t_{1}+t_{2}\right) / 2$ and $\tau=$ $t_{1}-t_{2}$ for three Landau excitations of duration $\tau_{\mathrm{e}}$ and energy $\omega_{\mathrm{e}}=3 / \tau_{\mathrm{e}}$ separated by $\Delta t=3 \tau_{\mathrm{e}}$. (b) Integral $\int \Delta \mathcal{G}_{S}^{(2 \mathrm{e})} \mathrm{d} \bar{t}$ i.e. average correlations as a function of $\tau$ only. Vanishing at $\tau=0$ is a manifestation of Pauli principle.

to $\Delta \mathcal{G}_{S}^{(2 \mathrm{e})}$. By partitioning electron and hole excitations emitted by the source against a reference Fermi sea at an electronic beam splitter, it dispatches the incoming coherences into the two outgoing channels numbered 1 and 2. Consequently, the outgoing interchannel two-electron coherence is related to $\Delta \mathcal{G}_{S}^{(2 \mathrm{e})}$ through

$$
\Delta \mathcal{G}_{\text {out }}^{(2 \mathrm{e})}\left(1, t_{1} ; 2, t_{2} \mid 1, t_{1}^{\prime} ; 2, t_{2}^{\prime}\right)=R T \Delta \mathcal{G}_{S}^{(2 \mathrm{e})}\left(t_{1}, t_{2} \mid t_{1}^{\prime}, t_{2}^{\prime}\right)
$$

where $R$ (resp. $T=1-R$ ) denote the reflexion (resp. transmission) probability of the QPC. As expected from quantum optics, $\Delta S_{12}^{\text {out }}\left(t, t^{\prime}\right)$ defined as the excess of current correlation

$$
S_{12}^{\text {out }}\left(t, t^{\prime}\right)=\left\langle i_{1}^{\text {out }}(t) i_{2}^{\text {out }}\left(t^{\prime}\right)\right\rangle-\left\langle i_{1}^{\text {out }}(t)\right\rangle\left\langle i_{2}^{\text {out }}\left(t^{\prime}\right)\right\rangle
$$

is related to the diagonal part of $\Delta \mathcal{G}_{S}^{(2 \mathrm{e})}$ by

$\Delta S_{12}^{\text {out }}\left(t, t^{\prime}\right)=\left(e v_{F}\right)^{2} R T \Delta \mathcal{G}_{S}^{(2 \mathrm{e})}\left(t, t^{\prime} \mid t, t^{\prime}\right)-\left\langle i_{1}(t)\right\rangle\left\langle i_{2}\left(t^{\prime}\right)\right\rangle$

thus showing that correlation measurements in the HBT setup give a direct access to the diagonal part of $\Delta \mathcal{G}_{S}^{(2 \mathrm{e})}$.

Let us now focus on the off-diagonal part of twoelectron coherence emitted by the source. First of all, for a train of electronic wavepackets, Eq. (6) shows that the only off-diagonal contributions to $\Delta \mathcal{G}_{S}^{(2 \mathrm{e})}$ arise from terms of the form $\varphi_{i}\left(t_{1}\right) \varphi_{j}\left(t_{2}\right) \varphi_{i}\left(t_{1}^{\prime}\right) \varphi_{j}\left(t_{2}^{\prime}\right)$ where $i \neq j$ and from their image under exchange operations $\left(t_{1}, t_{2} ; t_{1}^{\prime}, t_{2}^{\prime}\right) \mapsto\left(t_{2}, t_{1} ; t_{1}^{\prime}, t_{2}^{\prime}\right)$ or $\left(t_{1}, t_{2} ; t_{1}^{\prime}, t_{2}^{\prime}\right) \mapsto$ $\left(t_{2}, t_{1} ; t_{2}^{\prime}, t_{1}^{\prime}\right)$. Since the antisymmetry

$$
\begin{aligned}
\Delta \mathcal{G}_{S}^{(2 \mathrm{e})}\left(t_{1}, t_{2} \mid t_{1}^{\prime}, t_{2}^{\prime}\right) & =-\Delta \mathcal{G}_{S}^{(2 \mathrm{e})}\left(t_{2}, t_{1} \mid t_{1}^{\prime}, t_{2}^{\prime}\right) \\
& =-\Delta \mathcal{G}_{S}^{(2 \mathrm{e})}\left(t_{1}, t_{2} \mid t_{2}^{\prime}, t_{1}^{\prime}\right)
\end{aligned}
$$

is purely kinematical, the physical two-electron coherence time corresponds to the decay of $\varphi_{i}\left(t_{1}\right) \varphi_{i}\left(t_{1}^{\prime}\right) \varphi_{j}\left(t_{2}\right) \varphi_{j}\left(t_{2}^{\prime}\right)$ as a function of $\tau_{1}=t_{1}-t_{1}^{\prime}$ and $\tau_{2}=t_{2}-t_{2}^{\prime}$ and not from their images under the above exchange operations. Therefore, in this particular situation, the twoelectron coherence time is related to the durations of the wavepackets that govern the single electron coherence time scale.

On the other hand, quantum superposition of two time-shifted Slater determinants introduces a new time scale for two-electron coherence. The two-electron state $\left(\psi^{\dagger}\left[\varphi_{a}\right] \psi^{\dagger}\left[\varphi_{b}\right]|F\rangle+\psi^{\dagger}\left[\varphi_{c}\right] \psi^{\dagger}\left[\varphi_{d}\right]|F\rangle\right) / \sqrt{2}$ with mutually orthogonal wavepackets leads to

$$
\Delta \mathcal{G}^{(2 \mathrm{e})}\left(t_{1}, t_{2} \mid t_{1}^{\prime}, t_{2}^{\prime}\right)=\frac{1}{2} \sum_{\substack{\text { pairs } \\\{i j,\{k l\}}} \Phi_{i j}\left(t_{1}, t_{2}\right) \Phi_{k l}^{*}\left(t_{1}^{\prime}, t_{2}^{\prime}\right)
$$

Terms involving identical pairs correspond to Eq. (6) and their contributions are located close to the diagonal. Assuming that $\varphi_{c, d}$ are the time-shifted by $\Delta T_{\mathrm{tb}}$ of $\varphi_{a, b}$, terms with $\{i, j\} \neq\{k, l\}$ contribute to off-diagonal two-electron coherences, now extending over $\Delta T_{\mathrm{tb}}$. These coherences are a signature of the timebin entanglement [45, 46] of the quantum superposition of $\psi^{\dagger}\left[\varphi_{a}\right] \psi^{\dagger}\left[\varphi_{b}\right]|F\rangle$ and $\psi^{\dagger}\left[\varphi_{c}\right] \psi^{\dagger}\left[\varphi_{d}\right]|F\rangle$.

To be captured, quantum correlations contained in the off-diagonal parts $\left(t_{1} \neq t_{1}^{\prime}\right.$ and $\left.t_{2} \neq t_{2}^{\prime}\right)$ must be converted into measurable quantities. As discussed by Haack et al. 39], an ideal Mach-Zehnder interferometer (MZI) converts off-diagonal coherence in the time domain into an average current, i.e. diagonal coherence. This idea naturally leads to a Franson interferometer like setup [37] depicted on Fig. 3, in which MZI are added in each outgoing arm of a beam splitter. Such a setup was introduced in electron quantum optics to evidence twoparticle Aharonov-Bohm effect and entanglement generation [47. Here, we use it differently, namely as a measurement device, as originally proposed in photon quantum optics [37, 45, 46.

The current correlation between the two outgoing arms Lout and Rout picks up a magnetic flux dependence through the Aharonov-Bohm magnetic phases $\Phi_{L, R}$. The contributions depending on both magnetic fluxes, called twice Aharonov-Bohm terms, involve delocalization on both MZI and are the only one involving offdiagonal two-electron coherences in the time domain. They give access to the intrinsic two-electron coherence of the source since 


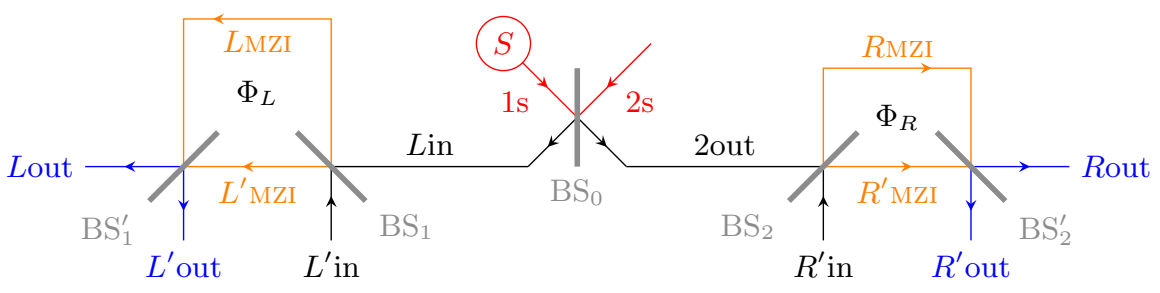

FIG. 3: (Color online) Franson interferometer setup used to access two-particle coherence in the time domain. The left (L) and right (R) MZI are pierced by magnetic fluxes $\Phi_{L, R}$ and have time delays $\delta t_{L, R}$. An electron source is connected to the channel 1s whereas channels $2 \mathrm{~s}, L^{\prime}$ in and $R^{\prime}$ in are grounded. The quantity of interest is the current correlation $\left\langle i_{L \text { out }}(t) i_{R \text { out }}\left(t^{\prime}\right)\right\rangle$.

$$
\begin{aligned}
\left\langle i_{L}\left(t_{1}\right) i_{R}\left(t_{2}\right)\right\rangle_{2 \mathrm{AB}} & =\left(e v_{F}\right)^{2} \kappa\left(\Delta \mathcal{G}_{S}^{(2 \mathrm{e})}\left(t_{1}-\delta t_{L}, t_{2}-\delta t_{R} \mid t_{1}, t_{2}\right) \mathrm{e}^{\mathrm{i}\left(\Phi_{L}+\Phi_{R}\right)}+\Delta \mathcal{G}_{S}^{(2 \mathrm{e})}\left(t_{1}, t_{2} \mid t_{1}-\delta t_{L}, t_{2}-\delta t_{R}\right) \mathrm{e}^{-\mathrm{i}\left(\Phi_{L}+\Phi_{R}\right)}\right. \\
& \left.+\Delta \mathcal{G}_{S}^{(2 \mathrm{e})}\left(t_{1}-\delta t_{L}, t_{2} \mid t_{1}, t_{2}-\delta t_{R}\right) \mathrm{e}^{\mathrm{i}\left(\Phi_{L}-\Phi_{R}\right)}+\Delta \mathcal{G}_{S}^{(2 \mathrm{e})}\left(t_{1}, t_{2}-\delta t_{R} \mid t_{1}-\delta t_{L}, t_{2}\right) \mathrm{e}^{-\mathrm{i}\left(\Phi_{L}-\Phi_{R}\right)}\right)
\end{aligned}
$$

where $\delta t_{L, R}$ are the time delays within the two MZI and $\kappa=R_{0} T_{0} R_{1} T_{1} R_{2} T_{2}$ denotes the product of all the reflexion and transmission probabilities of the beam splitters. Each contribution to the r.h.s. of Eq. (13) can be isolated by a Fourier transform of current correlation with respect to $\Phi_{L, R}$. Low frequency correlations thus gives access to the real and imaginary parts of the integral over $\left(t_{1}, t_{2}\right)$ of $\Delta \mathcal{G}_{S}^{(2 \mathrm{e})}\left(t_{1}-\delta t_{R}, t_{2}-\delta t_{L} \mid t_{1}, t_{2}\right)$.

Fig. $4 \mathrm{a}$-b present these quantities as functions of $\delta t_{L}$ and $\delta t_{R}$ when $(S)$ emits a pair of Landau excitations separated by $\Delta t=3 \tau_{\mathrm{e}}$. Both plots show a central peak at $\delta t_{L}=\delta t_{R} \simeq 0$ and satellite peaks at $\delta t_{L} \simeq-\delta t_{R} \simeq \pm 3 \tau_{\mathrm{e}}$. The central peak arises from single electron interferences which are visible as soon as $\delta t_{L}$ and $\delta t_{R}$ are shorter than the single electron coherence time. The satellite peaks are due to non-local two-electron interferences between the two time-shifted electrons of the pair. Their symmetry comes from the generic antisymmetry of two-electron coherence, Eq. (11). They spread along the line $\delta t_{L}=-\delta t_{R}$ over the total duration of the train corresponding to a classical correlation time and, since they do not depend on the pair's emission time, they are the same for a statistical mixture of two time shifted identical electron pairs. Note that the noise signal is of the order of the HOM signal for an HOM experiment $e^{2} f$ multiplied by $1 / 16$ due to the two MZI. This is of the order of $3 \times 10^{-30} \mathrm{~A}^{2} / \mathrm{Hz}$ for a $2 \mathrm{GHz}$ source, and therefore within reach of state of the art current noise measurements [15, 17.

For a superposition of time-shifted electron pairs, offdiagonal two-electron coherences appear in the expected signals [7] as shown Fig. 4-d. In this case, the twoelectron coherence time $\Delta T_{\mathrm{tb}}$ corresponds to the spreading along the $\delta t_{L}=\delta t_{R}$ diagonal. Considering interactions in the regime where electron/hole excitations generated by the incoming electron pair are well separated from the injected electronic excitations [48] shows that,
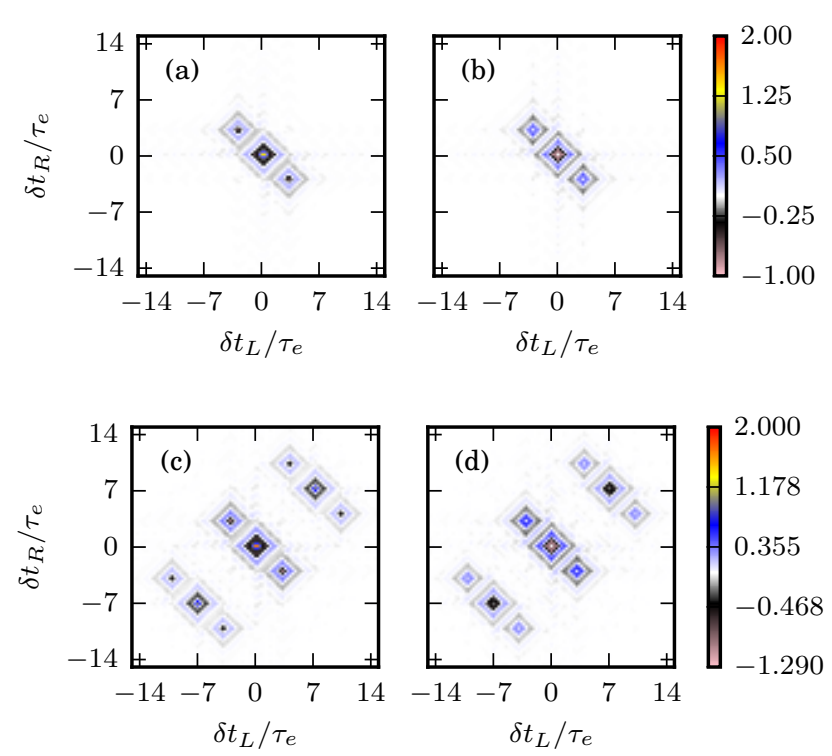

FIG. 4: (Color online) Density plots for real (panels a and c) and imaginary (panels b and d) parts of the averaged excess two-electron coherence as a function of $\left(\delta t_{L}, \delta t_{R}\right)$, extracted from the $\Phi_{L}+\Phi_{R}$ dependance of the low frequency correlation (13). Panels (a) and (b): signal for a pair of Landau excitations of duration $\tau_{\mathrm{e}}$ and energy $\omega_{\mathrm{e}}=3 / \tau_{\mathrm{e}}$ separated by $\Delta t=3 \tau_{\mathrm{e}}$. The plot is identical for a statistical mixture of two time-shifted identical electron pairs. Panels (c) and (d): signal expected for a quantum superposition of two such pairs in two time-bins, separated by $\Delta T_{\mathrm{tb}}=7 \tau_{\mathrm{e}}$.

for wavepackets of very short duration the weight of $\delta t_{L}=\delta t_{R}$ satellite peaks is decreased by an effective two electron decoherence arising from the overlap of the imprints left in the effective environnement by the two time-shifted electron pairs [49. The weight reduction of the $\delta t_{L}=\delta t_{R}$ satellite peaks with respect to the cen- 
tral peak is thus a direct measurement of two-electron decoherence [54].

To conclude, we have defined the intrinsic two-electron coherence emitted by an electronic source, related it to two-electron wavefunctions. We have shown how to access its off-diagonal part through current correlations in an ideal Franson interferometer and how it gives access to two-electron decoherence. The MZI being sensitive to decoherence, this protocol suffers from the same limitations as the proposal by Haack et al. 39] for single electron coherence but this problem can be circumvented by replacing MZI by devices converting off-diagonal single electron coherences into measurable quantities. For example, twoelectron correlations in energy could be accessed using energy filters [7], as put forward by Moskalets [25. Such measurements would also probe two electron relaxation and decoherence [49]. Correlations of finite frequency currents seem promising since interaction effects in the detection stage are encoded in finite frequency admittances [50]. Finally, in the same way Hanbury Brown and Twiss bypassed the challenges posed by amplitude interferomery by moving to intensity interferometry [51, two-electron coherences could be accessed through an appropriate measurement of correlations of current noise. This extends the idea of single electron tomography [2] to two-electron coherence. Measuring current noise correlations in mesoscopic conductors is notoriously difficult [52] but we think that this method deserves further investigation.

We thank M. Moskalets and Ch. Grenier for useful discussions. This work was supported by the ANR grants "1shot" (ANR-2010-BLANC-0412) and "1shot reloaded" (ANR-14-CE32-0017).

[1] E. Bocquillon, V. Freulon, F. Parmentier, J.M. Berroir, B. Plaçais, C. Wahl, J. Rech, T. Jonckheere, T. Martin, C. Grenier, et al., Ann. Phys. (Berlin) 526, 1 (2014).

[2] C. Grenier, R. Hervé, E. Bocquillon, F. Parmentier, B. Plaçais, J.M. Berroir, G. Fève, and P. Degiovanni, New Journal of Physics 13, 093007 (2011).

[3] G. Haack, M. Moskalets, and M. Büttiker, Phys. Rev. B 87201302 (2013).

[4] Y. Ji, Y. Chung, D. Sprinzak, M. Heiblum, D. Mahalu, and H. Shtrikman, Nature 422, 415 (2003).

[5] P. Roulleau, F. Portier, D.C. Glattli, P. Roche, A. Cavanna, G. Faini, U. Gennser, and D. Mailly, Phys. Rev. B 76, 161309 (2007).

[6] P. Roulleau, F. Portier, D.C. Glattli, P. Roche, A. Cavanna, G. Faini, U. Gennser, and D. Mailly, Phys. Rev. Lett. 100, 126802 (2008).

[7] C. Altimiras, H. le Sueur, U. Gennser, A. Cavanna, D. Mailly, and F. Pierre, Nature Physics 6, 34 (2010).

[8] H. le Sueur, C. Altimiras, U. Gennser, A. Cavanna, D. Mailly, and F. Pierre, Phys. Rev. Lett. 105, 056803 (2010).
[9] G. Fève, A. Mahé, J.M. Berroir, T. Kontos, B. Plaçais, D. Glattli, A. Cavanna, B. Etienne, and Y. Jin, Science 316, 1169 (2007).

[10] M. Blumenthal, B. Kaestner, L. Li, S. Giblin, T. Janssen, M. Pepper, D. Anderson, G. Jones, and D. Ritchie, Nature Physics 3, 343 (2007).

[11] S. Hermelin, S. Takada, M. Yamamoto, S. Tarucha, A. Wieck, L. Saminadayar, C. Bäerle, and T. Meunier, Nature 477, 435 (2011).

[12] F. Hohls, A. C. Welker, C. Leicht, L. Fricke, B. Kaestner, P. Mirovsky, A. Müller, K. Pierz, U. Siegner, and H. W. Schumacher, Phys. Rev. Lett. 109, 056802 (2012).

[13] J. Dubois, T. Jullien, F. Portier, P. Roche, A. Cavanna, Y. Jin, W. Wegscheider, P. Roulleau, and D. Glattli, Nature 502, 659 (2013).

[14] E. Bocquillon, F.D. Parmentier, C. Grenier, J.M. Berroir, P. Degiovanni, D.C. Glattli, B. Plaçais, A. Cavanna, Y. Jin, and G. Fève, Phys. Rev. Lett. 108, 196803 (2012).

[15] E. Bocquillon, V. Freulon, J.M. Berroir, P. Degiovanni, B. Plaçais, A. Cavanna, Y. Jin, and G. Fève, Science 339, 1054 (2013).

[16] A. I. Lvovsky and M. G. Raymer, Rev. Mod. Phys. 81, 299 (2009).

[17] T. Jullien, P. Roulleau, B. Roche, A. Cavanna, Y. Jin, and D. C. Glattli, Nature 514, 603 (2014).

[18] D. Ferraro, A. Feller, A. Ghibaudo, E. Thibierge, E. Bocquillon, G. Fève, C. Grenier, and P. Degiovanni, Phys. Rev. B 88, 205303 (2013).

[19] C. Wahl, J. Rech, T. Jonckheere, and T. Martin, Phys. Rev. Lett. 112, 046802 (2014).

[20] D. Ferraro, B. Roussel, C. Cabart, E. Thibierge, G. Fève, C. Grenier, and P. Degiovanni, Phys. Rev. Lett. 113, 166403 (2014).

[21] P.-A. Huynh, F. Portier, H. le Sueur, G. Faini, U. Gennser, D. Mailly, F. Pierre, W. Wegscheider, and P. Roche, Phys. Rev. Lett. 108, 256802 (2012).

[22] C. Altimiras, H. le Sueur, U. Gennser, A. Cavanna, D. Mailly, and F. Pierre, Phys. Rev. Lett. 105, 226804 (2010).

[23] J. Keeling, I. Klich, and L.S. Levitov, Phys. Rev. Lett. 97, 116403 (2006).

[24] J. Dubois, T. Jullien, C. Grenier, P. Degiovanni, P. Roulleau, and D. C. Glattli, Phys. Rev. B 88, 085301 (2013).

[25] M. Moskalets, Phys. Rev. B 89, 045402 (2014).

[26] R. Glauber, Phys. Rev. 130, 2529 (1963).

[27] S. Wölk, M. Huber, and O. Gühne, Phys. Rev. A 90, 022315 (2014).

[28] L. Amico, R. Fazio, A. Osterloh, and V. Vedral, Rev. Mod. Phys. 80, 517 (2008).

[29] J.P. Brantut, J. Meineke, D. Stadler, S. Krinner, and T. Esslinger, Science 337, 1069 (2012).

[30] S. Krinner, D. Stadler, D. Husmann, J.P. Brantut, and T. Esslinger, Nature 517, 64 (2015).

[31] R. Lopes, A. Imanaliev, A. Aspect, M. Cheneau, D. Boiron, and C.I. Westbrook, Nature 520, 66 (2015).

[32] P. Samuelsson, E.V. Sukhorukov, and M. Büttiker, Phys. Rev. Lett. 92, 026805 (2004).

[33] P. Samuelsson and M. Büttiker, Phys. Rev. B 73, 041305R (2006).

[34] I. Neder, N. Ofek, Y. Chung, M. Heiblum, D. Mahalu, and V. Umansky, Nature 448, 333 (2007).

[35] N. M. Chtchelkatchev, G. Blatter, G. B. Lesovik, and T. Martin, Phys. Rev. B 66, 161320 (2002).

[36] P. Samuelsson, E. V. Sukhorukov, and M. Büttiker, Phys. 
Rev. Lett. 91, 157002 (2003).

[37] J. D. Franson, Phys. Rev. Lett. 62, 2205 (1989).

[38] V. Giovannetti and K. Yuasa, Phys. Rev. B 86, 115429 (2012).

[39] G. Haack, M. Moskalets, J. Splettstoesser, and M. Büttiker, Phys. Rev. B 84, 081303 (2011).

[40] R. Glauber, Phys. Rev. Lett. 10, 84 (1962).

[41] C. Grenier, R. Hervé, G. Fève, and P. Degiovanni, Mod. Phys. Lett. B 25, 1053 (2011), proceedings of the Statphys 24 satellite meeting "International Conference on Frustrated Spin Systems, Cold Atoms and Nanomaterials" (Hanoi, 14-16 July 2010).

[42] A. Mahé, F.D. Parmentier, E. Bocquillon, J.M. Berroir, D.C. Glattli, T. Kontos, B. Plaçais, G. Fève, A. Cavanna, and Y. Jin, Phys. Rev. B 82, 201309 (2010).

[43] F.D. Parmentier, E. Bocquillon, J.M. Berroir, D.C. Glattli, B. Plaçais, G. Fève, M. Albert, C. Flindt, and M. Büttiker, Phys. Rev. B 85, 165438 (2012).

[44] R. Hanbury Brown and R. Twiss, Nature 177, 27 (1956).

[45] J. Brendel, N. Gisin, W. Tittel, and H. Zbinden, Phys. Rev. Lett. 82, 2594 (1999).

[46] I. Marcikic, H. de Riedmatten, W. Tittel, V. Scarani,
H. Zbinden, and N. Gisin, Phys. Rev. A 66, 062308 (2002).

[47] J. Splettstoesser, M. Moskalets, and M. Büttiker, Phys. Rev. Lett. 103, 076804 (2009).

[48] P. Degiovanni, C. Grenier and G. Fève, Phys. Rev. B 80, 241307(R) (2009).

[49] C. Cabart et al, (2016), In preparation.

[50] E. Bocquillon, V. Freulon, J.M. Berroir, P. Degiovanni, B. Plaçais, A. Cavanna, Y. Jin, and G. Fève, Nature Communications 4, 1839 (2013).

[51] R. Hanbury Brown and R. Twiss, Nature 178, 1046 (1956).

[52] E. Zakka-Bajjani, J. Dufouleur, N. Coulombel, P. Roche, D. C. Glattli, and F. Portier, Phys. Rev. Lett. 104, 206802 (2010).

[53] Landau excitations are described by a truncated Lorentzian in energy space: $\widetilde{\varphi}(\omega)=\mathcal{N}^{-1 / 2} \Theta(\omega) /(\omega-$ $\left.\omega_{\mathrm{e}}+\frac{\mathrm{i}}{2 \tau_{\mathrm{e}}}\right)$ where $\mathcal{N}$ is the normalisation constant.

[54] In practice, a small $\Delta T$ would be required for observing these peaks and a more quantitative analysis required. 